\title{
Supervisory Patterns and Practices: Case Studies at the University of Manchester, United Kingdom
}

\begin{abstract}
The student-supervisor relationship is vital in $\mathrm{PhD}$ process. Past research had shown that only highly unusual graduate students successfully completed their research degree programs if the relationship with their supervisors is poor. There are many factors that could contribute to poor achievement of foreign students and one of the most important critical factors found which contributed to this problem is the weaknesses in supervision they receive. This research is to identify the patterns of supervision employed by supervisors and the best appropriate practices. This study is using the case study method where three PhD students from three major disciplines of study namely arts, science and social science were involved in this research. As a result, the research had developed the best effective way of student and supervisor relationship. In order to have better relationship between the both parties, they have no doubt to be involved in more regular meetings or discussion process and to go through certain levels before, during and after each meeting. This should be practiced by lecturers, students and especially the foreign students in order to optimize the effectiveness of the student and supervisor's relationship.
\end{abstract}

Keyword: Foreign Student, Graduate Student, PhD, Supervision, Supervisory Practices 